\title{
Para-Quaternionic Structures on the 3-Jet Bundle
}

\author{
Bouazza Kacimi, Fouzi Hathout*, H.Mohamed Dida and Mokhtaria Barnoussi
}

(Communicated by Yusuf YAYLI)

\begin{abstract}
In this paper we construct an almost para-quaternionic structure on the 3-jet bundle of an almost parahermitian manifold and we study its integrability. We give a necessary and sufficient conditions that are provided for these structures to become para-hyper-Kähler and we prove that the 3 -jet bundle can not be a para-quaternionic Kähler manifold.

Keywords: Para-quaternionic; para-hyperhermitian; 3-jet bundle; $\lambda$-lift; diagonal metric.

AMS Subject Classification (2010): Primary 53A45; Secondary 53C15.

${ }^{*}$ Corresponding author
\end{abstract}

\section{Introduction}

A para-quaternionic structure on a manifold consists of an almost para hypercomplex structure which is a triple of endomorphisms of the tangent bundle $\mathbb{J}=\left\{J_{1}, J_{2}, J_{3}\right\}$, where $J_{1}$ is almost complex structure and $J_{2}, J_{3}$ are almost product structures satisfying anti-commutation relations and compatible with a semi-Riemannian metric necessarily of neutral signature. Moreover, if the structures $\mathbb{J}$ are parallel with respect to the Levi-Civita connection of the compatible metric, one arrives at the concept of para-hyper-Kähler structure and also named neutral hyperKähler or hypersymplectic structure ([3], [13], [14], [16], [18]...). The para-quaternionic and para-hyperhermitian structures are structures that appear in theorical physics, precisely, in string theory and integrable systems ([1], [9], [11]...).

On the other hand, the 3-jet bundle or the third order tangent bundle $T^{3} M$ of a smooth $n$-dimensional manifold $M$ is the $4 n$-dimensional smooth manifold of equivalent classes of curves $c$ on $M$ that agree up to their 3-velocity or the manifold of 3 -jet denoted $j^{3} c$. That is a generalization of the tangent bundle $T M$. This bundle has been studied with different names by many authors (see [5], [7]) for 2-jet bundle and generalized to the $r$-jet bundle in ([6]), where $T^{r} M$ is the smooth manifold of equivalent classes of curves $c$ on $M$ that agree up to their $r$-velocity or a manifold of $r$-jets.

Dodson and Radivoiovici prove that $T^{2} M$ becomes a vector bundle over $M$ with structure group $G L(2 n ; R)$ if the manifold $M$ is endowed with additional structure: a linear connection $\nabla$ ([7]), this result was generalized to $T^{r} M(r \geq 2)$ in ([6]). Then, the 3 -jet bundle of $n$-dimensional manifold $M$ is a vector bundle when $M$ is endowed with a linear connection $\nabla$.

The linear connection $\nabla$ on a manifold $M$ defines a diffeomorphism $S$ between the 3-jet bundle $T^{3} M$ and the Whitney sum of three copies of the tangent bundles TM. S is a fibre diffeomorphism of locally trivial bundle but it is not an isomorphism of natural bundles. Next, using the vertical and horizontal lift $\left(X^{V}, X^{H}\right)$ of vector fields $X \in \Gamma(T M)$ we define by the $\lambda$-lift the adapted frame $\left\{X^{0}, X^{1}, X^{2}, X^{3}\right\}$, so a sequence of distributions $E_{0}, E_{1}, E_{2}$ and $E_{3}$ on $T^{3} M$ such that $T\left(T^{3} M\right)=\underset{i=\overline{0,3}}{\oplus} E_{i}$, when $\lambda=1 . E_{0}, E_{1}$ coincide with $H$ and $V$ respectively the horizontal and the vertical subspaces of $T M$. The $\lambda$-lift of tensor fields on manifold $M$ to the 3 -jet bundle $T^{3} M$, is a generalization of vertical and horizontal lift of geometric structures to the tangent bundle $T M$ (see [6], [10]).

Received : 30-August-2016, Accepted : 06-November-2016 
The main purpose of this paper is to construct a para-quaternionic structure or para-hyperhermitian structure on the 3-jet bundle which is the generalization of this construction on tangent bundle (see [13], [20]), we also investigate its integrability, we obtain the necessary and sufficient conditions for these structures to become para-hyper-Kähler and finally we prove that the 3 -jet bundle can not be a para-quaternionic Kähler manifold.

\section{Preliminaries}

An almost product (resp. complex) structure on a smooth manifold $M$ is given by a tensor field $P$ (resp. $J$ ) of type $(1,1)$ on $M$ such that,

$$
P \neq \pm I d \text { and } P^{2}=I d \text {. (resp. } J^{2}=-I d \text { ) }
$$

$(M, P)$ (resp. $(M, J))$ is called an almost product (resp. complex) manifold. Moreover, if $M$ is endowed with pseudo-Riemannian metric $g$ satisfying

$$
g(P X, P Y)=-g(X, Y)(\text { resp. } g(J X, J Y)=g(X, Y))
$$

for all vector fields $X, Y$ on $M,(M, g, P)$ (resp. $(M, g, J))$ is called an almost para-hermitian (resp. hermitian) structure.

When $M$ support three tensor fields $\mathbb{J}=\left(J_{\alpha}\right)_{\alpha=1,2,3}$ where $J_{1}$ is an almost complex structure and $J_{2}, J_{3}$ are an almost product structures satisfying:

$$
\left\{\begin{array}{l}
J_{\alpha}^{2}=-\varepsilon_{\alpha} I d \\
J_{1} J_{2}=-J_{2} J_{1}=J_{3}
\end{array}\right.
$$

where $\alpha=1,2,3, \varepsilon_{1}=1, \varepsilon_{2}=\varepsilon_{3}=-1$, then $M$ is said to be an almost para-hypercomplex manifold and denoted $(M, \mathbb{J})$. Its dimension is multiple of 4 .

A semi-Riemannian metric $g$ on $(M, \mathbb{I})$ is said to be compatible or adapted to the almost para-hypercomplex structure $\mathbb{J}$ if it satisfies

$$
g\left(J_{1} X, J_{1} Y\right)=-g\left(J_{2} X, J_{2} Y\right)=-g\left(J_{3} X, J_{3} Y\right)=g(X, Y)
$$

for all vector fields $X, Y$ on $M$. The pair $(g, \mathbb{J})$ is called an almost para-hyperhermitian structure on $M$ and the triple $(M, g, \mathbb{J})$ is said to be an almost para-hyperhermitian manifold. Its adapted metric is of neutral signature $(2 n, 2 n)$. If $\mathbb{J}$ is parallel with respect to the Levi-Civita connection of $g$, then the manifold is called a para-hyper-Kähler.

Moreover, we say that $\mathbb{J}$ is integrable if its Nijenhuis tensor

$$
N_{\alpha}(X, Y)=\left[J_{\alpha} X, J_{\alpha} Y\right]-J_{\alpha}\left[X, J_{\alpha} Y\right]-J_{\alpha}\left[J_{\alpha} X, Y\right]+J_{\alpha}^{2}[X, Y] ; \alpha=1,2,3
$$

is zero for all vector fields $X$ and $Y$ on $M$, then $(M, \mathbb{J})$ is called a para-hypercomplex manifold. If $g$ is a semiRiemannian metric adapted to structure $\mathbb{J}$, then the pair $(g, \mathbb{J})$ is said to be a para-hyperhermitian structure on $M$ and $(M, g, \mathbb{J})$ is called a para-hyperhermitian manifold.

For a $n$-dimensional manifold $M$, let assume that there is a rank 3-sub bundle $\sigma$ of $\operatorname{End}(T M)$ such that a local basis $\left\{J_{1}, J_{2}, J_{3}\right\}$ of sections of $\sigma$ exists satisfying the formula (2.1). Then the bundle $\sigma$ is called a para-quaternionic structure on $M$ and $\left\{J_{1}, J_{2}, J_{3}\right\}$ is called a canonical local basis of $\sigma$. Moreover, $(M, \sigma)$ is said to be an almost para-quaternionic manifold.

A pseudo-Riemannian metric $g$ is said to be adapted to the para-quaternionic structure $\sigma$ if any local basis $\left\{J_{1}, J_{2}, J_{3}\right\}$ of $\sigma$ satisfies the formula (2.2). $(M, \sigma, g)$ is said to be an almost hermitian para-quaternionic manifold.

\section{Bundle of the 3-jet}

Let $M$ be an $n$-dimensional smooth differentiable manifold. For each $x \in M$, we define an equivalence relation on the set $C_{x}=\{c:(-\varepsilon, \varepsilon) \rightarrow M / c$ is smooth and $c(0)=x, \varepsilon>0\}$ by

$$
c \approx_{x} h \Longleftrightarrow c^{(i)}(0)=h^{(i)}(0) \text { for } i=\overline{1,3},
$$

where $c^{(i)}$ denote the derivation of order $i$ of $c$ :

$$
c^{(i)}:(-\varepsilon, \varepsilon) \rightarrow T M ; t \rightarrow\left[\frac{d c^{(i)}(t)}{d t^{(i)}}\right](0)
$$


Definition 3.1. The 3-jet space or the third order tangent space of $M$ at the point $x$ denoted by $T_{x}^{3} M$ is the quotient $C_{x} / \approx_{x}$ and the 3 -jet-bundle or the third order tangent bundle of $M$ is the union of all the 3 -jets spaces

$$
T^{3} M=\underset{x \in M}{\cup} T_{x}^{3} M
$$

We denote by $j_{x}^{3} c$ the equivalence class of $c$ with respect to $\approx_{x}$ and by $j^{3} c$ an element of $T^{3} M$.

Moreover, when $M$ is endowed with a linear connection, $T^{3} M$ becomes a vector bundle with structure group the general linear group $G L(2 n ; \mathbb{R})$ and $(3+1) n$-dimensional smooth manifold.

Now, let $\nabla$ be a linear connection on $M$. Let $\pi_{3}: T^{3} M \rightarrow M$ be the projection defined by $\pi_{3}\left(j^{3} c\right)=c(0)$, if $\left(U, x^{1}, \ldots, x^{n}\right)$ is a chart on $M$, then we consider the induced chart $\left(\pi_{3}^{-1}(U), x^{i, \lambda}\right)_{i=\overline{1, n}, \lambda=\overline{0,3}}$ on $T^{3} M$ defined by

$$
x^{i, \lambda}\left([c]_{3}\right)=\frac{1}{\lambda !} \frac{d^{\lambda}}{d t^{\lambda}}\left(x^{i} \circ c\right)(0) .
$$

Using the connection $\nabla$ we can define the diffeomorphism $S$ by

$$
\begin{aligned}
& S \quad: \quad T^{3} M \rightarrow T M+T M+T M \\
& S\left([c]_{3}\right)=\left(\dot{c}(0),\left(\nabla_{c} \dot{c}\right)(0),\left(\nabla_{c} \nabla_{c} \dot{c}\right)(0)\right)
\end{aligned}
$$

$\nabla_{c}$ denotes the covariant derivation along $c$ and $c$ is the velocity vector field of $c$, with

$$
\nabla_{c} \cdot \dot{c}=\left(\frac{d^{2} c^{i}}{d t^{2}}+p_{1}^{i}\right) \frac{\partial}{\partial x^{i}} \text { and } \nabla_{c} \nabla_{c} \cdot \dot{i}=\left(\frac{d^{3} c^{i}}{d t^{3}}+p_{2}^{i}\right) \frac{\partial}{\partial x^{i}}
$$

where $p_{1}^{i}$ (resp. $p_{2}^{i}$ ) is a polynomial of degree one (resp. two) on $\frac{d^{k} c^{j}}{d t^{k}}$ with $k \leq 1$ (resp. $k \leq 2$ ) and the coefficients of $p_{1}^{i}$ (resp. $p_{2}^{i}$ ) depend on the connection coefficients $\Gamma_{j k}^{i}$ (resp. $D_{\alpha} \Gamma_{j k}^{i}$, with $|\alpha| \leq 1$ ).

\section{Lift from $M$ to $T^{3} M$}

Let $(M, g)$ be a pseudo-Riemannian manifold, $\nabla$ it's Levi-Civita connection and $R$ it's curvature tensor.

\section{Some results of the lift from $M$ to $T M$}

Let be $f$ a function on $M$. For any vector field $X$ on $M$, we denote by $f^{V}$ the vertical lift of $f$ to $T M$ defined by

$$
f^{V}=f \circ \pi ; \pi \text { is projection from } T M \text { to } M
$$

Let be $X$ a vector field on $M$. Then there is one and only one vector field $X^{V}$ on $T M$ called the vertical lift of $X$ such that

$$
X^{V}\left(f^{V}\right)=0, \text { for every } f
$$

The connection $\nabla$ define a horizontal distribution $H$ on $T M$ such that

$$
T(T M)=V \oplus H \quad \text { where } V=\operatorname{ker} d \pi
$$

Since for every point $z$ of $T M$

$$
d_{z} \pi_{/ H_{z}}: H_{z} \rightarrow T_{\pi(z)} M
$$

is an isomorphism, then, if $X$ is a vector field on $M$, we can define

$$
X^{H}(z)=\left(d_{z} \pi_{/ H_{z}}\right)^{-1}\left(X_{\pi(z)}\right)
$$

$X^{H}$ is a vector field on $T M$ called the horizontal lift of $X$ to $T M$.

Consequently, $\left\{X^{H}, X^{V}\right\}$ is a $2 n$-frame which is called the adapted frame to $\nabla$ in $T M$. 


\section{Lift from $M$ to $T^{3} M$}

Let $X \in \Gamma(T M)$ be a vector field on $M$. For $\lambda=\overline{0,3}$, the $\lambda$-lift of $X$ to $T^{3} M$ is defined by

$$
\begin{aligned}
& X^{0}=S_{*}^{-1}\left(X^{H}, X^{H}, X^{H}\right) \\
& X^{1}=S_{*}^{-1}\left(X^{V}, 0,0\right) \\
& X^{2}=S_{*}^{-1}\left(0, X^{V}, 0\right) \\
& X^{3}=S_{*}^{-1}\left(0,0, X^{V}\right)
\end{aligned}
$$

when $\lambda=1$ the $\lambda$-lift of $\left(X^{0}, X^{1}\right)$ coincide with $\left(X^{H}, X^{V}\right)$ in $T M$. If $\lambda=2$, the $\lambda$-lift was studied in ([5]) and for any $\lambda \geq 1$, it was studied in ([6]). $\left\{X^{\lambda}\right\}_{\lambda=0, . ., 3}$ is a $4 n$-frame called the adapted frame in $T^{3} M$.

Proposition 4.1. For all vector fields $X, Y \in \Gamma(T M)$ and $p \in T^{3} M$, we have the identities

$$
\begin{aligned}
{\left[X^{0}, Y^{0}\right]_{p} } & =[X, Y]_{p}^{0}-\left((R(X, Y) u)^{1}+(R(X, Y) w)^{2}+(R(X, Y) z)^{3}\right) \\
{\left[X^{0}, Y^{i}\right] } & =\left(\nabla_{X} Y\right)^{i} \\
{\left[X^{i}, Y^{0}\right] } & =-\left(\nabla_{Y} X\right)^{i} \\
{\left[X^{i}, Y^{j}\right] } & =0
\end{aligned}
$$

where $(u, w, z)=S(p)$ and $i, j=\overline{1,3}$.

Proof. For proof see [5] and [6].

Definition 4.1. The diagonal lift of $g$ to $T^{3} M$ denoted by ${ }^{D} g$ is defined by

$$
\left\{\begin{array}{l}
\text { i) }{ }^{D} g\left(X^{i}, Y^{i}\right)=g(X, Y) \\
\text { ii) }{ }^{D} g\left(X^{i}, Y^{j}\right)=0
\end{array}\right.
$$

for $i, j=\overline{0,3}(i \neq j)$ and $X, Y$ vector fields in $T M .{ }^{D} g$ coincide with Sasaki metric on $T M$. (see [6])

The Levi-Civita connection ${ }^{D} \nabla$ of ${ }^{D} g$ is given by Koszul formula as following

$$
\begin{aligned}
& \left({ }^{D} \nabla_{X^{0}} Y^{0}\right)_{p}=\left(\nabla_{X} Y\right)_{p}^{0}-\frac{1}{2}\left((R(X, Y) u)^{1}+(R(X, Y) w)^{2}+(R(X, Y) z)^{3}\right) \\
& \left({ }^{D} \nabla_{X^{0}} Y^{i}\right)_{p}=\left(\nabla_{X} Y\right)_{p}^{i}+\frac{1}{2}(R(X, Y) u)^{0} \\
& \left({ }^{D} \nabla_{X^{i}} Y^{0}\right)_{p}=\frac{1}{2}(R(X, Y) u)^{0} \\
& \left({ }^{D} \nabla_{X^{i}} Y^{j}\right)_{p}=0 \text { for } i, j \neq 0
\end{aligned}
$$

for all vector fields $X, Y$ in $T M, p \in T^{3} M$ and $(u, w, z)=S(p)$.

Now, we suppose for sections 5 and 6 that $(M, P, g)$ be an almost para-hermitian $n$-dimensional manifold.

\section{Para-hyperhermitian structures}

Definition 5.1. We define three tensor fields $\widetilde{\mathbb{J}}=\left(J_{\alpha}\right)_{\alpha=1,2,3}$ on $T^{3} M$ by the equalities:

$$
\left\{\begin{array}{c}
J_{1} X^{0}=X^{2} \\
J_{1} X^{1}=X^{3} \\
J_{1} X^{2}=-X^{0} \\
J_{1} X^{3}=-X^{1}
\end{array},\left\{\begin{array}{c}
J_{2} X^{0}=P X^{2} \\
J_{2} X^{1}=P X^{3} \\
J_{2} X^{2}=P X^{0} \\
J_{2} X^{3}=P X^{1}
\end{array},\left\{\begin{array}{c}
J_{3} X^{0}=P X^{0} \\
J_{3} X^{1}=P X^{1} \\
J_{3} X^{2}=-P X^{2} \\
J_{3} X^{3}=-P X^{3}
\end{array}\right.\right.\right.
$$

Then we have the theorem

Theorem 5.1. The 3-jet bundle $T^{3} M$ admits an almost para-hypercomplex structure $\widetilde{\mathbb{J}}$ which is a para-hyperhermitian with respect to ${ }^{D} g$. 
Proof. From the definition (5.1), we have for $J_{1}$

$\left\{\begin{array}{c}J_{1}^{2} X^{0}=J_{1} X^{2}=-X^{0} \\ J_{1}^{2} X^{1}=J_{1} X^{3}=-X^{1} \\ J_{1}^{2} X^{2}=-J_{1} X^{0}=-X^{2} \\ J_{1}^{2} X^{3}=-J_{1} X^{1}=-X^{3}\end{array} \Longrightarrow J_{1}^{2} \tilde{X}=-\tilde{X}\right.$

the calculations for $J_{2}^{2}$ and $J_{3}^{2}$ are analogous to $J_{1}^{2}$.

For the anti-commutation rules, we have

$\left\{\begin{array}{rl}J_{2} J_{1} X^{0}=J_{2} X^{2} & =P X^{0}=J_{3} X^{0} \\ J_{2} J_{1} X^{1}=J_{2} X^{3} & =P X^{1}=J_{3} X^{1} \\ J_{2} J_{1} X^{2}=-J_{2} X^{0} & =-P X^{2}=J_{3} X^{2} \\ J_{2} J_{1} X^{3}=-J_{2} X^{1} & =-P X^{3}=J_{3} X^{3}\end{array} \Longrightarrow J_{2} \tilde{X}=J_{3} \tilde{X}\right.$.

Its similar for $-J_{1} J_{2}=J_{3}$, thus

$$
J_{1}^{2}=-J_{2}^{2}=-J_{3}^{2}=-I d \text { and } J_{2} J_{1}=-J_{1} J_{2}=J_{3}
$$

Using the definition (5.1) for $J_{1}$, we have

$$
\begin{aligned}
& \left\{\begin{array} { l } 
{ D _ { g } g ( J _ { 1 } X ^ { 0 } , J _ { 1 } Y ^ { 0 } ) = D ^ { D } g ( X ^ { 0 } , Y ^ { 0 } ) } \\
{ D g ( J _ { 1 } X ^ { 0 } , J _ { 1 } Y ^ { 1 } ) = 0 = D g ( X ^ { 0 } , Y ^ { 1 } ) } \\
{ D g ( J _ { 1 } X ^ { 0 } , J _ { 1 } Y ^ { 2 } ) = 0 = D ^ { D } g ( X ^ { 0 } , Y ^ { 2 } ) } \\
{ D g ( J _ { 1 } X ^ { 0 } , J _ { 1 } Y ^ { 3 } ) = 0 = D g ( X ^ { 0 } , Y ^ { 3 } ) } \\
{ D g ( J _ { 1 } X ^ { 1 } , J _ { 1 } Y ^ { 1 } ) = { } ^ { D } g ( X ^ { 1 } , Y ^ { 1 } ) }
\end{array} \text { and } \quad \left\{\begin{array}{l}
{ }^{D} g\left(J_{1} X^{1}, J_{1} Y^{2}\right)=0={ }^{D} g\left(X^{1}, Y^{2}\right) \\
D g\left(J_{1} X^{1}, J_{1} Y^{3}\right)=0=D g\left(X^{1}, Y^{3}\right) \\
D g\left(J_{1} X^{2}, J_{1} Y^{2}\right)={ }^{D} g\left(X^{2}, Y^{2}\right) \\
D g\left(J_{1} X^{2}, J_{1} Y^{3}\right)=0=D g\left(X^{2}, Y^{3}\right) \\
D g\left(J_{1} X^{3}, J_{1} Y^{3}\right)=0=D^{D} g\left(X^{3}, Y^{3}\right)
\end{array}\right.\right. \\
& { }^{D} g\left(J_{1} \tilde{X}, J_{1} \tilde{Y}\right)={ }^{D} g(\tilde{X}, \tilde{Y}) .
\end{aligned}
$$

And similarly for $J_{2}$ and $J_{3}$, we get the compatibility of $\widetilde{\mathbb{J}}$ with $g^{D}$ defined in formula (4.2)

$$
{ }^{D} g\left(J_{1} \widetilde{X}, J_{1} \tilde{Y}\right)=-{ }^{D} g\left(J_{2} \widetilde{X}, J_{2} \tilde{Y}\right)=-{ }^{D} g\left(J_{3} \widetilde{X}, J_{3} \widetilde{Y}\right)={ }^{D} g(\widetilde{X}, \widetilde{Y}) .
$$

Thus, $\widetilde{\mathbb{J}}$ is a para-hyperhermitian structure with respect to ${ }^{D} g$.

\section{Study of integrability}

First of all, we mention a general proposition:

Proposition 6.1. Let $(M, g, P)$ be an almost product manifold, then we have

$$
\begin{aligned}
& 1 /[P X, P Y]=\left(\nabla_{P X} P\right)(Y)-\left(\nabla_{P Y} P\right)(X)+P\left(\nabla_{P X} Y-\nabla_{P Y} X\right) \\
& 2 / P[P X, Y]=P \nabla_{P X} Y-P \nabla_{Y} P X \\
& 3 / P[X, P Y]=P \nabla_{X} P Y-P \nabla_{P Y} X
\end{aligned}
$$

for all vector fields $X, Y$ on $M$.

The integrability of structure $\widetilde{\mathbb{J}}$ is given by 16 equations for each $j, i=\overline{0,3}$, in following proposition.

Proposition 6.2. The Nijenhuis tensor of structure $J_{1}$ is given by

$$
\begin{aligned}
N_{1}\left(X^{0}, Y^{0}\right) & =(R(X, Y) u)^{1}+(R(X, Y) v)^{2}+(R(X, Y) w)^{3} \\
N_{1}\left(X^{2}, Y^{2}\right) & =(R(X, Y) u)^{1}+(R(X, Y) v)^{2}+(R(X, Y) w)^{3} \\
N_{1}\left(X^{2}, Y^{0}\right) & =N_{1}\left(X^{2}, Y^{0}\right) \\
& =J_{1}\left((R(X, Y) u)^{1}+(R(X, Y) v)^{2}+(R(X, Y) w)^{3}\right) \\
& =(R(X, Y) u)^{3}-(R(X, Y) v)^{0}-(R(X, Y) w)^{1} \\
N_{1}\left(X^{i}, Y^{j}\right) & =\text { ofor all } i, j=\overline{0,3} \text { and }(i, j) \neq\{(0,0),(0,2),(2,0),(2,2)\}
\end{aligned}
$$


Similarly, we deduce for $J_{2}$

$$
\begin{aligned}
N_{2}\left(X^{0}, Y^{0}\right)= & -\left(P\left(\nabla_{X} P\right) Y\right)^{0}+(R(X, Y) u)^{1}+(R(X, Y) v)^{2}+(R(X, Y) w)^{3} \\
N_{2}\left(X^{0}, Y^{2}\right)= & -\left(P\left(\nabla_{X} P\right) Y+\left(\nabla_{P Y} P\right) Y\right)^{2} \\
& +(R(X, P Y) u)^{3}+(R(X, P Y) v)^{0}+(R(X, P Y) w)^{1} \\
N_{2}\left(X^{2}, Y^{0}\right)= & \left(P\left(\nabla_{Y} P\right) X+\left(\nabla_{P X} P\right) X\right)^{2} \\
& +(R(P X, Y) u)^{3}+(R(P X, Y) v)^{0}+(R(P X, Y) w)^{1} \\
N_{2}\left(X^{0}, Y^{i}\right)= & \left(P\left(\nabla_{X} P\right) Y\right)^{i} \text { for } i=1,3 \\
N_{2}\left(X^{i}, Y^{0}\right)= & -\left(P\left(\nabla_{Y} P\right) X\right)^{i} \text { for } i=1,3 \\
N_{2}\left(X^{i}, Y^{j}\right)= & 0 \text { for } i, j=1,3 \\
N_{2}\left(X^{1}, Y^{2}\right)= & -\left(\left(\nabla_{P Y} P\right) X\right)^{3} \\
N_{2}\left(X^{2}, Y^{1}\right)= & \left(\left(\nabla_{P X} P\right) Y\right)^{3} \\
N_{2}\left(X^{2}, Y^{2}\right)= & \left(\left(\nabla_{P X} P\right) Y-\left(\nabla_{P Y} P\right) X\right)^{0} \\
& -(R(P X, P Y) u)^{1}+(R(P X, P Y) v)^{2}+(R(P X, P Y) w)^{3} \\
N_{2}\left(X^{2}, Y^{3}\right)= & \left(\left(\nabla_{P X} P\right) Y\right)^{1} \\
N_{2}\left(X^{3}, Y^{2}\right)= & -\left(\left(\nabla_{P Y} P\right) X\right)^{1}
\end{aligned}
$$

and finally for $J_{3}$

$$
\begin{aligned}
N_{3}\left(X^{0}, Y^{0}\right)= & \left(\left(\nabla_{P X} P\right) Y-\left(\nabla_{P Y} P\right) X-P\left(\nabla_{X} P\right) Y+P\left(\nabla_{Y} P X\right)\right)^{0}+ \\
& +(P(R(P X, Y) u)+P(R(X, P Y) u)-R(P X, P Y) u-R(X, Y) u)^{1} \\
& +(P(R(P X, Y) v)+P(R(X, P Y) v)-R(P X, P Y) v-R(X, Y) v)^{2} \\
& +(P(R(P X, Y) w)+P(R(X, P Y) w)-R(P X, P Y) w-R(X, Y) w)^{3} \\
& \\
N_{3}\left(X^{0}, Y^{1}\right)= & \left(\nabla_{P X} P Y-P\left(\nabla_{X} P\right) Y-P \nabla_{P X} Y\right)^{1} \\
& =\left(\left(\nabla_{P X} P\right) Y-P\left(\nabla_{X} P\right) Y\right)^{1} \\
N_{3}\left(X^{1}, Y^{0}\right)= & -\left(\nabla_{P Y} P X-P\left(\nabla_{Y} P\right) X-P \nabla_{P Y} X\right)^{1} \\
& =\left(P\left(\nabla_{Y} P\right) X-\left(\nabla_{P Y} P\right) X\right)^{1} \\
N_{3}\left(X^{0}, Y^{i}\right)= & \left(P \nabla_{P X} Y-P\left(\nabla_{X} P\right) Y-\nabla_{P X} P Y\right)^{i} \\
& =-\left(P\left(\nabla_{X} P\right) Y+\left(\nabla_{P X} P\right) Y\right)^{i} \text { for } i=2,3 \\
N_{3}\left(X^{i}, Y^{0}\right)= & -\left(P \nabla_{P Y} X-P\left(\nabla_{Y} P\right) X-\nabla_{P Y} P X\right)^{i} \\
= & \left(P\left(\nabla_{Y} P\right) X+\left(\nabla_{P Y} P\right) X\right)^{i} \text { for } i=2,3 \\
N_{3}\left(X^{i}, Y^{j}\right)= & 0 \text { for } i, j=\overline{1,3}
\end{aligned}
$$

for all $X, Y$ vector fields on $M$ and $S(p)=(u, w, z)$.

Proof. We recall that the Nijenhuis tensor of the structures $\widetilde{\mathbb{J}}=\left(J_{\alpha}\right)_{\alpha=1,2,3}$ is

$$
\begin{aligned}
N_{\alpha}\left(X^{i}, Y^{j}\right) & =\left[J_{\alpha} X^{i}, J_{\alpha} Y^{j}\right]-J_{\alpha}\left[X^{i}, J_{\alpha} Y^{j}\right]-J_{\alpha}\left[J_{\alpha} X^{i}, Y^{j}\right]+J_{\alpha}^{2}\left[X^{i}, Y^{j}\right] \\
\text { for } \alpha & =1,2,3 \text { and } j, i=\overline{0,3} .
\end{aligned}
$$

Using the definition (5.1) and the formula (4.3), we get the Nijenhuis tensor of $J_{1}$ (i.e $N_{1}$ ). For $N_{2}$ and $N_{3}$, we use also the proposition (6.1).

Then, we have the following theorem

Theorem 6.1. The structure $\widetilde{\mathbb{J}}=\left(J_{\alpha}\right)_{\alpha=\overline{1,3}}$ is an almost para-hypercomplex structure on $T^{3} M$ which becomes almost para-hyperhermitian with respect to the diagonal lift metric ${ }^{D} \mathrm{~g}$. Moreover, $\left(T^{3} M,{ }^{D} \mathrm{~g}, \widetilde{\mathbb{J}}\right.$ ) is para-hyperhermitian (i.e $\widetilde{\mathbb{J}}$ is integrable) if and only if $(M, P, g)$ is a flat para-Kähler manifold. 


\section{Para-quaternionic structures}

Let $(M, \sigma, g)$ be an almost hermitian para-quaternionic $4 n$-dimensional manifold. We can locally choose a para-hypercomplex structure $\mathbb{J}=\left\{J_{1}, J_{2}, J_{3}\right\}$ which is a basis of $\sigma$. In fact, by the definition an almost hermitian para-quaternionic manifold is locally almost hermitian para-hypercomplex.

We can locally define a nondegenerate 2 -forms

$$
\Omega_{J_{\alpha}}(X, Y)=g\left(X, J_{\alpha} Y\right), \alpha=1,2,3
$$

However, the 4 -form

$$
\Omega=\Omega_{J_{1}} \wedge \Omega_{J_{1}}-\Omega_{J_{2}} \wedge \Omega_{J_{2}}-\Omega_{J_{3}} \wedge \Omega_{J_{3}}
$$

is defined globally on $M$.

Definition 7.1. An almost hermitian para-hypercomplex $4 n$-dimensional manifold $(M, g, \mathbb{J})(n \geq 1)$ is parahyperKähler if $\nabla \mathbb{J}=0$ (i.e $\nabla J_{\alpha}=0, \alpha=\overline{1,3}$ ), where $\nabla$ is the Levi-Civita connection with respect to the metric $g$. An almost hermitian para-quaternionic $4 n$-dimensional manifold $(M, \sigma, g)(n \geq 2)$ is called para-quaternionic Kähler manifold if $\nabla \Omega=0$ and $M$ is not para-hyperKähler. (see [17],[13],[19])

Let $(M, P, g)$ be an almost para-hermitian $n$-dimensional manifold. We shall call an almost para-quaternionic structure on $T^{3} M$ any sub bundle $\tilde{\sigma}$ of the vector bundle $\operatorname{End}\left(T^{3} M\right)$, locally spanned by a para-hyperhermitian structure $\widetilde{\mathbb{J}}$. The pair $\left(T^{3} M, \tilde{\sigma}\right)$ will be called an almost para-quaternionic manifold and $\left(T^{3} M, \tilde{\sigma},^{D} g\right)$ is called an almost hermitian para-quaternionic manifold.

We define three nondegenerate 2 -forms in $\left(T^{3} M, \tilde{\sigma},{ }^{D} g\right)$ by

$$
\tilde{\Omega}_{J_{\alpha}}(\widetilde{X}, \widetilde{Y})={ }^{D} g\left(\widetilde{X}, J_{\alpha} \widetilde{Y}\right), \alpha=\overline{1,3}
$$

for any vector fields $\widetilde{X}$ and $\widetilde{Y}$ in $T^{3} M$. The 4 -form is given by

$$
\tilde{\Omega}=\tilde{\Omega}_{J_{1}} \wedge \tilde{\Omega}_{J_{1}}-\tilde{\Omega}_{J_{2}} \wedge \tilde{\Omega}_{J_{2}}-\tilde{\Omega}_{J_{3}} \wedge \tilde{\Omega}_{J_{3}}
$$

Proposition 7.1. The Levi-Civita connection of ${ }^{D} g$ satisfies ${ }^{D} \nabla \tilde{\Omega}=0$ if and only if $(M, P, g)$ is a flat para-Kähler manifold.

In order to prove the proposition, first, we need the following lemma.

Lemma 7.1. $\quad$ i. From the formulas (4.2), (7.1) and the definition (5.1), the three 2 -forms $\tilde{\Omega}_{J_{\alpha}}$ are given by

$$
\begin{aligned}
& \left\{\begin{array}{l}
\tilde{\Omega}_{J_{1}}\left(X^{i}, Y^{j}\right)=g(X, Y) \text { for }(i, j)=(2,0) \text { and }(3,1) \\
\tilde{\Omega}_{J_{1}}\left(X^{i}, Y^{j}\right)=-g(X, Y) \text { for }(i, j)=(0,2) \text { and }(1,3) \\
\tilde{\Omega}_{J_{1}}\left(X^{i}, Y^{j}\right)=0 \text { for the remaining cases }(i, j)
\end{array}\right. \\
& \left\{\begin{array}{l}
\tilde{\Omega}_{J_{2}}\left(X^{i}, Y^{j}\right)=g(X, P Y) \text { for }(i, j)=(2,0),(3,1),(0,2) \text { and }(1,3) \\
\tilde{\Omega}_{J_{2}}\left(X^{i}, Y^{j}\right)=0 \text { for the remaining cases }(i, j)
\end{array}\right. \\
& \left\{\begin{array}{l}
\tilde{\Omega}_{J_{3}}\left(X^{i}, Y^{j}\right)=g(X, P Y) \text { for }(i, j)=(0,0),(1,1) \\
\tilde{\Omega}_{J_{3}}\left(X^{i}, Y^{j}\right)=-g(X, P Y) \text { for }(i, j)=(2,2),(3,3) \\
\tilde{\Omega}_{J_{3}}\left(X^{i}, Y^{j}\right)=0 \text { for the remaining cases }(i, j)
\end{array}\right.
\end{aligned}
$$

with $i, j=\overline{0,3}$ and for any vector fields $X, Y$ in $T M$.

ii.

$$
\begin{aligned}
{ }^{D} \nabla_{U^{i}}\left(\tilde{\Omega}_{J_{\alpha}} \wedge \tilde{\Omega}_{J_{\alpha}}\right)\left(X^{i}, Y^{j}, Z^{k}, W^{l}\right)= & 2 \sum_{Y^{j}, Z^{k}, W^{l}}\left({ }^{D} g\left(X^{i},\left({ }^{D} \nabla_{U^{i}} J_{\alpha}\right) Y^{j}\right) \tilde{\Omega}_{J_{\alpha}}\left(Z^{k}, W^{l}\right)\right. \\
& \left.+\tilde{\Omega}_{J_{\alpha}}\left(X^{i}, Y^{j}\right){ }^{D} g\left(Z^{k},\left({ }^{D} \nabla_{U^{i}} J_{\alpha}\right) W^{l}\right)\right)
\end{aligned}
$$

where the sum is taken over cyclic permutations of $Y^{j}, Z^{k}, W^{l}$ and $i, j, k, l=\overline{0,3}$. 
iii.

$$
\left({ }^{D} \nabla_{X^{i}} J_{\alpha}\right) Y^{j}={ }^{D} \nabla_{X^{i}}\left(J_{\alpha} Y^{j}\right)-J_{\alpha}\left({ }^{D} \nabla_{X^{i}} Y^{j}\right)
$$

Using the formulas (4.3) and (7.3), we have

$$
\begin{aligned}
\left({ }^{D} \nabla_{X^{0}} J_{\alpha}\right) Y^{0}= & { }^{D} \nabla_{X^{0}}\left(J_{\alpha} Y^{0}\right)-J_{\alpha}\left(\nabla_{X} Y\right)^{0} \\
& +\frac{1}{2} J_{\alpha}\left((R(X, Y) u)^{1}+(R(X, Y) w)^{2}+(R(X, Y) z)^{3}\right) \\
\left({ }^{D} \nabla_{X^{0}} J_{\alpha}\right) Y^{i}= & { }^{D} \nabla_{X^{0}}\left(J_{\alpha} Y^{j}\right)-J_{\alpha}\left(\nabla_{X} Y\right)^{i}+\frac{1}{2} J_{\alpha}(R(u, Y) X)^{0} \\
\left({ }^{D} \nabla_{X^{i}} J_{\alpha}\right) Y^{0}= & { }^{D} \nabla_{X^{i}}\left(J_{\alpha} Y^{0}\right)+\frac{1}{2} J_{\alpha}(R(X, Y) u)^{0} \\
\left({ }^{D} \nabla_{X^{i}} J_{\alpha}\right) Y^{j}= & { }^{D} \nabla_{X^{i}}\left(J_{\alpha} Y^{j}\right) \text { for } i, j=\overline{1,3}
\end{aligned}
$$

Proof of the proposition 10. ${ }^{D} \nabla_{X^{i}} \tilde{\Omega}$ is given by $4^{4}=256$ identities when the indices $i, j, k, l$ varies from 0 to 3 . For this, we have used a computer program with matlab software. ${ }^{D} \nabla_{X^{i}} \tilde{\Omega}$ is calculated in three parts as follows

$$
\begin{aligned}
{ }^{D} \nabla_{X^{i}} \tilde{\Omega} & ={ }^{D} \nabla_{X^{i}}\left(\tilde{\Omega}_{J_{1}} \wedge \tilde{\Omega}_{J_{1}}\right)-{ }^{D} \nabla_{X^{i}}\left(\tilde{\Omega}_{J_{2}} \wedge \tilde{\Omega}_{J_{2}}\right)-{ }^{D} \nabla_{X^{i}}\left(\tilde{\Omega}_{J_{3}} \wedge \tilde{\Omega}_{J_{3}}\right) \\
& =I_{1}-I_{2}-I_{3} \quad i=\overline{0,3}
\end{aligned}
$$

Taking account of lemma (7.1)-(i,ii) as databases of computer program, each part $I_{\alpha}(\alpha=1,2,3)$ is calculated in $(256)$ identities when the indices $i, j, k, l$ vary from 0 to 3 . We remark that all (256) identities are a sum of terms of types

$$
g(X, Y){ }^{D} g\left(Z^{k},\left({ }^{D} \nabla_{X^{i}} J_{\alpha}\right) W^{l}\right) \text { and } g(X, P Y){ }^{D} g\left(Z^{k},\left({ }^{D} \nabla_{X^{i}} J_{\alpha}\right) W^{l}\right)
$$

where $X, Y, Z, W$ commutes over cyclic permutations except $X$ in ${ }^{D} \nabla_{X^{i}}$.

However, from the lemma (7.1) (iii), we have for the structure $J_{1}$

$$
\begin{aligned}
& \left({ }^{D} \nabla_{X^{0}} J_{1}\right) Y^{0}=\frac{1}{2}\left((R(X, Y) u)^{3}-(R(X, Y) z)^{1}\right) \\
& \left.\left({ }^{D} \nabla_{X^{0}} J_{1}\right) Y^{1}=\frac{1}{2}\left((R(u, Y) X)^{0}+(R(u, Y) X)^{2}\right)\right) \\
& \left({ }^{D} \nabla_{X^{0}} J_{1}\right) Y^{2}=\frac{1}{2}\left((R(X, Y) u)^{1}+2(R(X, Y) w)^{2}+(R(X, Y) z)^{3}\right) \\
& \left({ }^{D} \nabla_{X^{0}} J_{1}\right) Y^{3}=\frac{1}{2}\left((R(u, Y) X)^{2}-(R(u, Y) X)^{0}\right) \\
& \left({ }^{D} \nabla_{X^{i}} J_{1}\right) Y^{0}=\frac{1}{2}(R(u, X) Y)^{2} \text { for } i=\overline{1,3} \\
& \left({ }^{D} \nabla_{X^{2}} J_{1}\right) Y^{2}=-\frac{1}{2}(R(u, X) Y)^{0} \\
& \left({ }^{D} \nabla_{X^{i}} J_{1}\right) Y^{j}=0 \text { for } i, j=1,3
\end{aligned}
$$

and for $J_{2}$

$$
\begin{aligned}
\left({ }^{D} \nabla_{X^{0}} J_{2}\right) Y^{0}= & \left(\left(\nabla_{X} P\right) Y\right)^{2}+\frac{1}{2}\left((R(u, P Y) X)^{0}\right. \\
& \left.+(P R(X, Y) w)^{0}+(P R(X, Y) z)^{1}+(P R(X, Y) u)^{3}\right) \\
\left({ }^{D} \nabla_{X^{0}} J_{2}\right) Y^{1}= & \left(\left(\nabla_{X} P\right) Y\right)^{3}+\frac{1}{2}\left((R(u, P Y) X)^{0}+(P R(u, Y) X)^{2}\right) \\
\left({ }^{D} \nabla_{X^{0}} J_{2}\right) Y^{2}= & \left.\left(\nabla_{X} P\right) Y\right)^{0}-\frac{1}{2}\left((R(X, P Y) u)^{1}+\left((R(P X, Y) w)^{2}\right.\right. \\
& \left.-(P R(u, Y) X))^{2}+(R(P X, Y) z)^{3}\right) \\
\left({ }^{D} \nabla_{X^{0}} J_{2}\right) Y^{3}= & \left.\left(\nabla_{X} P\right) Y\right)^{1}+\frac{1}{2}\left((R(u, P Y) X)^{0}+(P R(u, Y) X)^{2}\right) \\
\left({ }^{D} \nabla_{X^{i}} J_{2}\right) Y^{0}= & \frac{1}{2}(P R(X, Y) u)^{2} \text { for } i=\overline{1,3} \\
\left({ }^{D} \nabla_{X^{i}} J_{2}\right) Y^{2}= & \frac{1}{2}(R(u, X) P Y)^{0} \text { for } i=\overline{1,3} \\
\left({ }^{D} \nabla_{X^{i}} J_{2}\right) Y^{j}= & 0 \text { for } i=1,2,3 \text { and } j=1,3
\end{aligned}
$$


finally for $J_{3}$

$$
\begin{aligned}
\left({ }^{D} \nabla_{X^{0}} J_{3}\right) Y^{0}= & \left(\left(\nabla_{X} P\right) Y\right)^{0}-\frac{1}{2}\left((R(X, P Y) u)^{1}+(R(X, P Y) w)^{2}+\right. \\
& \left.(R(X, P Y) z)^{3}\right)+\left(P(R(X, Y) u)^{1}-P(R(X, Y) w)^{2}\right. \\
& \left.-P(R(X, Y) z)^{3}\right) \\
\left({ }^{D} \nabla_{X^{0}} J_{3}\right) Y^{1}= & \left(\left(\nabla_{X} P\right) Y\right)^{1}+\frac{1}{2}(P R(u, Y) X)^{0} \\
\left({ }^{D} \nabla_{X^{0}} J_{3}\right) Y^{2}= & -\left(\left(\nabla_{X} P\right) Y\right)^{2}+\frac{1}{2}(P R(u, Y) X)^{0} \\
\left({ }^{D} \nabla_{X^{0}} J_{3}\right) Y^{3}= & -\left(\left(\nabla_{X} P\right) Y\right)^{3}+\frac{1}{2}(P R(u, Y) X)^{0} \\
\left({ }^{D} \nabla_{X^{i}} J_{3}\right) Y^{0}= & \frac{1}{2}(R(u, X) P Y+P(R(X, Y) u))^{0} \text { for } i=\overline{1,3} \\
\left({ }^{D} \nabla_{X^{i}} J_{\alpha}\right) Y^{j}= & 0 \text { for } i, j=\overline{1,3}
\end{aligned}
$$

Taking into account the formulas (7.7), (7.8) and (7.9), the terms (7.6) vanishes if and only if $P$ is parallel and without curvature (i.e $\nabla P=0$ and $R \equiv 0)$. Then, ${ }^{D} \nabla_{X^{i}} \tilde{\Omega}$ vanishes if and only if $(M, P, g)$ is a flat para-Kähler manifold.

Remark 7.1. If $P$ is not parallel or $R \neq 0$ then ${ }^{D} \nabla_{X^{i}} \tilde{\Omega} \neq 0$.

Finally, we have the following theorems

Theorem 7.1. Let $\left(T^{3} M,^{D} \mathrm{~g}\right)$ be the 3-jet bundle with para-hyperhermitian structure $\widetilde{\mathbb{J}}$ with respect to the diagonal lift metric $g^{D} .\left(T^{3} M,{ }^{D} g\right)$ is para-hyperKähler manifold if and only if $(M, P, g)$ is a flat para-Kähler manifold (i.e $\nabla P=0$ and $R \equiv 0$ ).

Proof. The proof is given from the formulas (7.7), (7.8) and (7.9).

Theorem 7.2. The almost hermitian para-quaternionic manifold $\left(T^{3} M, \tilde{\sigma}{ }^{D} \mathrm{~g}\right)$ is never para-quaternionic Kähler manifold.

Proof. From the proposition (7.1), we have ${ }^{D} \nabla \tilde{\Omega}=0$ if $(M, P, g)$ is a flat para-Kähler manifold i.e $\nabla P=0$ and $R \equiv 0$ or in this case, $\left(T^{3} M, \tilde{\sigma},^{D} g\right)$ is para-hyper Kähler manifold (i.e $\left.{ }^{D} \nabla \widetilde{\mathbb{J}}=0\right)$ and taking account about the definition (7.1), $\left(T^{3} M, \tilde{\sigma}{ }^{D} g\right)$ is never para-quaternionic Kähler manifold.

A possible extension of this paper is to construct a para-hyperhermitian (quaternionic) structures on $r$-jet bundle with $r=-1 \bmod [4]$ as a naturally generalization of the tangent bundle of an almost para-hermitian manifold.

\section{Acknowledgement}

The authors would like to express their deep thanks to Prof. Dr. Arif Salimov for his useful suggestions and to the referee for his/her remarks.

\section{References}

[1] Barret, J., Gibbons, G.W., Perry, M.J., Pope, C.N. and Ruback P., Kleinian geometry and the $N=2$ superstring, Int . J. Mod. Phys. A 9(1994), 1457-1494.

[2] Bielawski, R., Complexification and hypercomplexification of manifolds with a linear connection, Internat. J. Math. 14(2003), no.8, 813-824.

[3] Dancer, A. and Swann, A., Hypersymplectic manifolds, in "Recent developments in pseudo-Riemannian geometry". ESI Lectures in Mathematics and Physics (2008), 97-148.

[4] Davidov, J., Grantcharov, G., Mushkarov, O. and Yotov, M., Para-hyperhermitian surfaces. Bull. Math. Soc. Sci. Math. Roumanie (N.S.) 52(2009), no.3, 281-289.

[5] Dida, H.M., Hathout, F. and Djaa, M., On the geometry of the second order tangent bundle with the diagonal lift metric, Int. Journal of Math. Anal. 3(2009), 443-456. 
[6] Djaa, M. and Gancarzewicz, J., The geometry of tangent bundles of order r, Boletin Academia Galega de Ciencias spain 4(1985), 147-165.

[7] Dodson, C.T.J. and Radivoiovici, M.S., Tangent and Frames Bundles of order two, Analele stiintifice ale Univesitatii "AL. I Gusa" 28(1982), 63-71.

[8] Dombrowski, P., On the geometry of the tangent bundle. J.Reine Angew. Math. 210(1962), 73-88.

[9] Feix, B., Hyperkahler metrics on cotangent bundles, J. Reine Angew. Math 532(2001), 33-46.

[10] Hathout, F. , Dida, H.M., Diagonal lift in the tangent bundle of order two and its applications, Turk. J. Math. 29(2005), 1-12.

[11] Hull, C.M., Actions for (2,1) sigma models and strings, Nucl. Phys. B 509(1998), 252-272.

[12] Ivanov, S., Sanov, V.T. and Zamkovoy, S., Hyper-para-Hermitian manifolds with torsion, J. Geom. Phys. 56(2006), no.4, 670-690.

[13] Ivanov, S. and Zamkovoy, S., Para-hermitianand para-quaternionic manifolds. Differ. Geom. Appl. 23(2005), 205-234.

[14] Kaledin, D., A canonical hyperkähler metric on the total space of a cotangent bundle. Proceedings of the Second Meeting "Quaternionic structures in mathematics and physics" (Rome, 1999), World Sci. Publishing, River Edge, NJ (2001), 195-230.

[15] Kamada, H., Neutral hyper-Kähler structures on primary Kodaira surfaces, Tsukuba J. Math. 23(1999) 321-332.

[16] Lanus, S. and Vîlcu, G.E., Some constructions of almost para-hyperhermitian structures on manifolds and tangent bundles, Int. J. Geom. Methods Mod. Phys., 5(2008), no.6, 893-903.

[17] Lonescu, A.M. and Vîlcu, G.E., A Note on para-quaternionic Manifolds, Missouri J. Math. Sci., 19(2007), $213-218$.

[18] Nakashima, Y. and Watanabe, Y., Some constructions of almost Hermitian and quaternion metric structures, Math. J. Toyama Univ., 13(1900), 119-138.

[19] Tahara, M., Marchiafava, S. and Watanabe, Y., Quaternionic Kähler structures on the tangent bundle of a complex space form, Rend. Ist. Mat. Univ. Trieste, 31(1999), 163-175.

[20] Vîlcu, G.E., Para-hyperhermitian structures on tangent bundles, Proceedings of the Estonian Academy of Sciences 60(2011), no.3, 165-173.

\section{Affiliations}

BOUAZZA KACIMI

Address: Mascara University 29000 Algeria.

E-MAIL: kacimibouazza@yahoo.fr

FOUZI HATHOUT

ADDRESS: Department of Mathematics, Faculty of Science, Saïda University 20000 AlgERIA.

E-MAIL: f.hathout@gmail.com, fouzi.hathout@univ-saida.dz

H.MOHAMED DiDA

AdDress: Department of Mathematics, Faculty of Science, Saïda University 20000 Algeria.

E-MAIL: mhamou.dida@gmail.com

MOKHTARIA BARNOUSSI

ADDRESS: MELT laboratory, Tlemcen University 13000 ALGERIA.

E-MAIL: mokhtaria.barnoussi@yahoo.fr 